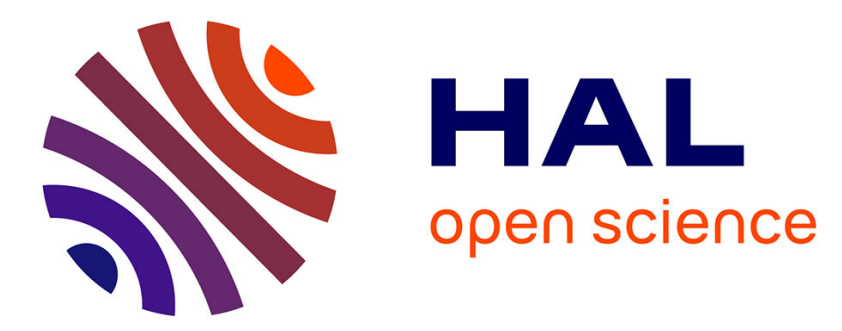

\title{
Investigating the gas phase emitter effect of caesium and cerium in ceramic metal halide lamps in dependence on the operating frequency
}

C Ruhrmann, M Westermeier, A Bergner, G M J F Luijks, P Awakowicz, J Mentel

\section{To cite this version:}

C Ruhrmann, M Westermeier, A Bergner, G M J F Luijks, P Awakowicz, et al.. Investigating the gas phase emitter effect of caesium and cerium in ceramic metal halide lamps in dependence on the operating frequency. Journal of Physics D: Applied Physics, 2011, 44 (35), pp.355202. 10.1088/0022$3727 / 44 / 35 / 355202$. hal-00649055

\section{HAL Id: hal-00649055 https://hal.science/hal-00649055}

Submitted on 7 Dec 2011

HAL is a multi-disciplinary open access archive for the deposit and dissemination of scientific research documents, whether they are published or not. The documents may come from teaching and research institutions in France or abroad, or from public or private research centers.
L'archive ouverte pluridisciplinaire HAL, est destinée au dépôt et à la diffusion de documents scientifiques de niveau recherche, publiés ou non, émanant des établissements d'enseignement et de recherche français ou étrangers, des laboratoires publics ou privés. 


\title{
Investigating the gas phase emitter effect of caesium and cerium in ceramic metal halide lamps in dependence on the operating frequency
}

\author{
C Ruhrmann ${ }^{1}$, M Westermeier ${ }^{1,2}$, A Bergner ${ }^{1}$, \\ G M J F Luijks ${ }^{3}, \mathrm{P}$ Awakowicz $^{1}$ and $\mathrm{J} \mathrm{Mentel}^{1}$ \\ ${ }^{1}$ Ruhr University Bochum, Electrical Engineering and Plasma Technology, D-44780 \\ Bochum, Germany \\ 2 Present address: RWE Effizienz GmbH, Freistuhl 7, D-44137 Dortmund, Germany \\ 3 Philips Lighting, GBU HID, P.O.box 80020, 5600JM Eindhoven, The Netherlands \\ E-mail: juergen.mentel@ruhr-uni-bochum.de
}

\begin{abstract}
The work function and with it the temperature of tungsten electrodes in HID lamps can be lowered and the lifetime of lamps increased by the gas phase emitter effect. A determination of the emitter effect of $C s$ and $C e$ is performed by phase resolved measurements of the electrode tip temperature $T_{t i p}(\varphi)$, plasma temperature $T_{p l}(\varphi)$ and particle densities $N(\varphi)$ by means of pyrometric, optical emission and broadband absorption spectroscopy in dependence on the operating frequency. The investigated HID lamps are ceramic metal halide lamps with transparent discharge vessels made of YAG, filled with a buffer gas consisting of $\mathrm{Ar}, \mathrm{Kr}$ and predominantly $\mathrm{Hg}$ and seeded with $\mathrm{CsI}$ or $\mathrm{CeI}_{3}$. In the YAG lamp seeded with $\mathrm{CsI}$ and $\mathrm{CeI}_{3}$ as well as in a YAG lamp seeded with $D y I_{3}$ (corresponding results can be found in a preceding paper) a gas phase emitter effect is observed in the cathodic phase due to a $\mathrm{Cs}, \mathrm{Ce}$ or $D y$ ion current. In the YAG lamp seeded with $C s I$ the phase averaged coverage of the electrode surface with emitter atoms decreases and the electrode temperature rises with increasing frequency, whereas the emitter effect of $C e$ and $D y$ is extended to the anodic phase, which leads to a decreased average temperature $T_{t i p}(\varphi)$ with increasing frequency. This different behaviour of the averaged values of $T_{t i p}(\varphi)$ for increasing frequency is caused by the differing adsorption energies $E_{a}$ of the respective emitter materials. In spite of the influence of $E_{a}$ on the coverage of the electrode with emitter atoms, the cathodic gas phase emitter effect produces in the YAG lamps seeded with $C s I, \mathrm{CeI}_{3}$ and $\mathrm{DyI}_{3}$ a general reduction of the electrode tip temperature $T_{\text {tip }}(\varphi)$ in comparison to a YAG lamp with $\mathrm{Hg}$ filling only.
\end{abstract}

Submitted to: J. Phys. D: Appl. Phys. 
Investigating the gas phase emitter effect of $C s$ and Ce in ceramic metal halide lamps 2

\section{Introduction}

Ceramic metal halide lamps offer presently the cheapest option to realize a point shaped light source with an output of more than one k-lumen satisfying the requirements of an efficacy of more than $100 \mathrm{~lm} / \mathrm{W}$, a life time of more than $10000 \mathrm{~h}$ and a colour rendering index higher than $90 \mathrm{cri}$. But the high thermal load of the electrodes, resulting in a high electrode temperature, is a quite serious problem with this special version of HID lamps.

The electrode temperature, both of the cathode and of the anode, is mainly determined by the work function of the electrode material $[1,2,3,4,5,6,7,8]$. If pure tungsten electrodes are used the temperature of the electrode tip is raised up to its melting point, $T_{m}=3695 \mathrm{~K}$, to supply a sufficiently high thermionic electron emission for a diffuse attachment of the lamp arc on the electrodes $[9,10,11,12,13,14,15]$. But the work function and with it the electrode temperature can be substantially reduced if a dipole layer consisting of a monolayer of atoms being electropositive with respect to tungsten is deposited on the tungsten surface. It lowers the potential barrier at the electrode surface for electrons leaving or entering the electrode.

Several techniques are applied to establish this so called emitter effect in HID lamps by a monolayer of electropositive atoms [16]. Doping tungsten with $\mathrm{ThO}_{2}$ is the most traditional method to cover the electrode surface with a dipole layer of thorium atoms dated from Langmuir [17]. According to the experience in vacuum electronics it is assumed that the monolayer is formed by the diffusion of thorium from the interior of the electrode, after dissociation of $\mathrm{ThO}_{2}$ into $\mathrm{Th}$ metal and oxygen [18]. But this simple view is doubtful in the case of HID lamp electrodes since detailed investigations on thoriated electrodes have revealed that only HID cathodes show an emitter effect $[2,19]$ but not HID anodes [6, 7]. Another method to reduce the work function is realised by depositing emitter material within the interspaces of a closely wounded tungsten coil ending up in some distance from the electrode tip. When the electrode is heated up the emitter material, e.g. barium, diffuses along the electrode surface to the tip [20, 21].

The most versatile approach is the gas phase emitter effect, in which case the monolayer is deposited from the gas phase. The number of pure metals, which are appropriate to realize a gas phase emitter effect in HID lamps, is rather low. But the number of elements can be considerably extended, if the high vapour pressure of metal iodides is employed, e.g. that of rare earth iodides, to implement a gas phase emitter effect. In some cases a gas phase emitter effect is clearly realized, both at the cathode and at the anode, e.g. in high pressure sodium lamps, if appropriate lamp parameters are chosen $[3,8]$. In other cases it may be confined to the cathode, at least if it is operated with a dc current. This situation seems to be present in a YAG lamp being seeded with $\mathrm{DyI}_{3}$ and different other additives as $\mathrm{NaI}$ and $\mathrm{TlI}$ [22]. If HID lamps, which are seeded with $\mathrm{ThI}_{4}$ [23], DyI $[24,25]$ or other metal iodides are operated with an ac 
or switched-dc current, it could not be decided definitely, whether the emitter effect is limited to the cathodic phase or also being effective within the anodic phase. To clarify this point, the gas phase emitter effect of $D y$ is investigated in a preceding paper [26] by spectroscopic measurements in front of the electrode and pyrometric measurements along the electrode rod in dependence on the operating frequency. These measurements have shown that the reduction of the electrode temperature is correlated to a high $D y$ ion density in front of the electrode within the cathodic phase, suggesting that the $D y$ monolayer is essentially sustained by a $D y$ ion current towards the cathode. But at higher operating frequencies a reduction of the work function is also effective within the anodic phase, as is shown by the reduction of the electrode temperature with increasing operating frequency. It may be attributed to the finite life time of the layer reflecting the adsorption energy of $D y$ on the tungsten surface of $E_{a}=4.97 \mathrm{eV}$.

If the hypothesis is valid that the atomic monolayer is mainly generated by an ion current towards the cathode then the gas phase emitter effect does not only depend on the reduction of the work function $\Delta \phi$ and the adsorption energy $E_{a}$ of the atoms within the dipole layer but also on the ionisation energy $E_{i}$ of the emitter material and the mobility $\mu_{i} \propto M_{i}^{-1}$ of the ions, on the electrode polarity and with it on the operating frequency. The reduction of the work function of tungsten $\phi_{W}$ may vary between $\Delta \phi=0$ and $\Delta \phi=\phi_{W}-\phi_{\text {emitter }}+\delta \phi$ where $\phi_{\text {emitter }}$ is the work function of a surface consisting of many layers of emitter material and $\delta \phi$ represents a reduction of the work function in the order of several hundred meV if the coverage $\Theta$ of the tungsten surface is incomplete $(0.4<\Theta<1)$ [27].

To clarify the role of the ion current the emitter effect of the alkali metal caesium and of the rare earth metal cerium are compared by measurements in YAG lamps performed at operating frequencies being varied from $1 \mathrm{~Hz}$ to $1 \mathrm{kHz}$. The buffer gas of the YAG lamps consisting of $A r, K r$ and predominantly $H g$ is seeded either with $C s I$ or $\mathrm{CeI}_{3}$. Caesium is characterized by a work function $\phi_{C s}=1.8 \mathrm{eV}$ and an ionisation energy $E_{i}=3.89 \mathrm{eV}$, which is very favourable for the gas phase emitter effect, but also by a quite low adsorption energy $E_{a}=3.37 \mathrm{eV}$ [28], which is counterproductive. Cerium

\begin{tabular}{c|c|c|c|c} 
& work function & ionisation energy & adsorption energy & relative mass \\
& $\phi$ & $E_{i}$ & $E_{a}$ & $A_{r}$ \\
\hline$C s$ & $1.8 \mathrm{eV}$ & $3.89 \mathrm{eV}$ & $3.37 \mathrm{eV}$ & 132.90 \\
$C e$ & $2.7 \mathrm{eV}$ & $5.54 \mathrm{eV}$ & $6.84 \mathrm{eV}$ & 140.12 \\
$D y$ & $3.1 \mathrm{eV}$ & $5.92 \mathrm{eV}$ & $4.97 \mathrm{eV}$ & 162.50 \\
$T l$ & $3.7 \mathrm{eV}$ & $6.11 \mathrm{eV}$ & $3.28 \mathrm{eV}$ & 204.38
\end{tabular}

Table 1: Values for work function $\phi$, ionisation energy $E_{i}$, adsorption energy $E_{a}$ on a tungsten surface and relative mass $A_{r}$ for $C s, C e, D y$ and $T l[28,29,30]$ 
is characterized by $\phi_{C e}=2.7 \mathrm{eV}, E_{i}=5.54 \mathrm{eV}$, values, which are less favourable than those of $C s$ but comparable with those of $D y$, and $E_{a}=6.84 \mathrm{eV}[29,30]$, which is nearly by a factor of two higher than the adsorption energy of $C s$ and also higher than the adsorption energy of $D y$. Since the relative masses of caesium $\left(A_{r}=132.9\right)$ and cerium $\left(A_{r}=140.12\right)$ are not very different, it may be assumed, that the mobility of the two ions is quite similar. Both a cerium ion current and a caesium ion current may generate within the cathodic phase a distinct emitter effect, but as a consequence of the different adsorption energies the life time of the $C e$ monolayer may be longer and therefore the dependence of the gas phase emitter effect of cerium on the operating frequency more pronounced than that of caesium. The data of $\phi, E_{i}, E_{a}$ and $A_{r}$ of $C s, C e$ and of $D y$, $T l$, by which the lamp was seeded in the preceding paper [26], are summarized in table 1.

To check this speculation the electrode temperature within the YAG lamps and the densities of the emitter materials in front of the YAG lamp electrodes are investigated in dependence on the operating frequency. In the case of the YAG lamp seeded with CsI the same measuring methods as in [26] are applied. The electrode temperature as well as the plasma temperature and the $C s$ atom density are determined by emission spectroscopy. The $C s$ ion density can not be measured since an appropriate $C s$ ion line is not available. But it is calculated taking into account that LTE is realized. In the case of the YAG lamp seeded with $\mathrm{CeI}_{3}$ for the electrode temperature as well as for the $\mathrm{Ce}$ atom density new measuring methods are applied, which give more reliable results than the previous ones. The $2 \mathrm{D}$ distribution of the electrode temperature is recorded by a photograph with a CCD camera, which is calibrated for the infrared wavelength of a narrowband interference filter in front of the camera [22]. The $C e$ atom density is determined by absorption spectroscopy. For this purpose the measuring system for emission spectroscopy is supplemented by an intense back light, realized by an UHP lamp $[20,21,31]$.

\section{Experimental set up}

\subsection{YAG lamp}

The lamps under investigation are special research HID lamps with simple rod-type pure tungsten electrodes with a diameter of $0.36 \mathrm{~mm}$, a length of $5 \mathrm{~mm}$, an inter electrode spacing of $7 \mathrm{~mm}$ and an ovoid-shaped discharge vessel made of yttrium alumina garnet $\left(Y_{6} A_{5} \mathrm{O}_{12}\right)$ shortened YAG [22]. YAG is a transparent ceramic and therefore suitable for optical observations. The YAG vessel is placed inside a sealed and evacuated outer bulb, made of fused silica doped with lead, for protection from UV light, mechanical protection and thermal insulation of the inner bulb. The discharge vessel is filled with a buffer gas, which consists of an argon-krypton-mixture $(30 \mathrm{kPa})$, and with $6 \mathrm{mg}$ mercury to generate a background pressure of $2 \mathrm{MPa}$ during lamp operation being required for a sufficiently high power input into the arc. $1 \mathrm{mg}$ of the respective emitter element $(C s I$, 
Investigating the gas phase emitter effect of $C s$ and Ce in ceramic metal halide lamps 5

$\left.\mathrm{CeI}_{3}, \mathrm{Dy} \mathrm{I}_{3}, \mathrm{TlI}\right)$ is added to the lamp filling to generate a gas phase emitter effect. A lamp without any emitter material is used as reference. The results which are obtained by the lamp seeded with $D y I_{3}$ and the reference lamp are already published in [26] (figure 3-5 and figure 1-2).

A sketch of the inner tube of a YAG lamp is shown in figure 1 while the filling properties of all YAG lamps presented in this work and in the preceding paper consulted for comparison [26] are listed in table 2.

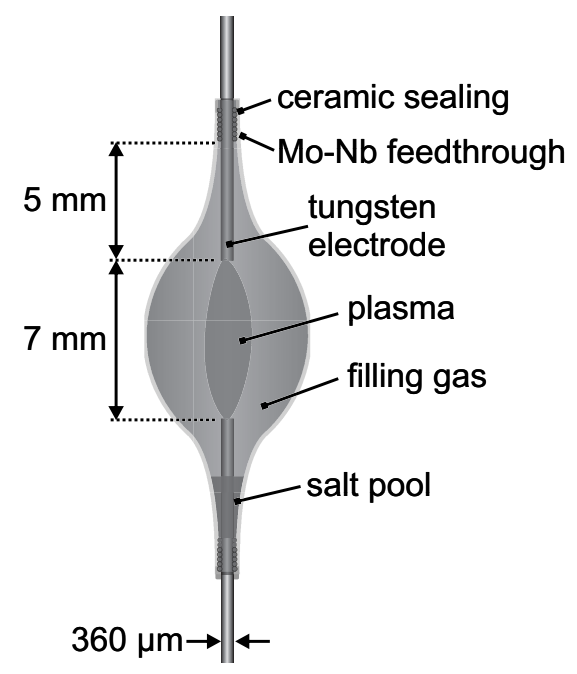

Figure 1: Schematic drawing of the inner tube of a YAG lamp

\begin{tabular}{c|c|c|c|c|c|c} 
Lamp & $\begin{array}{c}H g \\
\text { type }\end{array}$ & $\begin{array}{c}\text { Total salt } \\
(\mathrm{mg})\end{array}$ & $\begin{array}{c}C s I \\
(\mathrm{mg})\end{array}$ & $\begin{array}{c}C e I_{3} \\
(\mathrm{~mol} \%)\end{array}$ & $\begin{array}{c}D y I_{3} \\
(\mathrm{~mol} \%)\end{array}$ & $\begin{array}{c}T l I \\
(\mathrm{~mol} \%)\end{array}$ \\
\hline $\mathrm{Hg}$ & 6 & 0 & 0 & 0 & 0 & 0 \\
$\mathrm{Cs}$ & 6 & 1 & 100 & 0 & 0 & 0 \\
$\mathrm{Ce}$ & 6 & 1 & 0 & 100 & 0 & 0 \\
$\mathrm{Dy}$ & 6 & 1 & 0 & 0 & 100 & 0 \\
$\mathrm{Tl} / \mathrm{Dy}$ & 6 & 2 & 0 & 0 & 50 & 50
\end{tabular}

Table 2: Properties of the filling of YAG lamps presented in this work and in the preceding paper consulted for comparison [26]

The YAG lamps, built for a nominal input power of $70 \mathrm{~W}$, are operated in vertical position. Since the accumulation of the liquid salt at the bottom of the discharge vessel hampers optical observations, all optical measurements are performed at the upper electrode. The investigated lamps are operated with switched-dc current at $0.8 \mathrm{~A}$ and frequencies of $1,10,100,500 \mathrm{~Hz}$ and $1 \mathrm{kHz}$ by a voltage controlled current source (Feucht 
Investigating the gas phase emitter effect of $C s$ and Ce in ceramic metal halide lamps 6

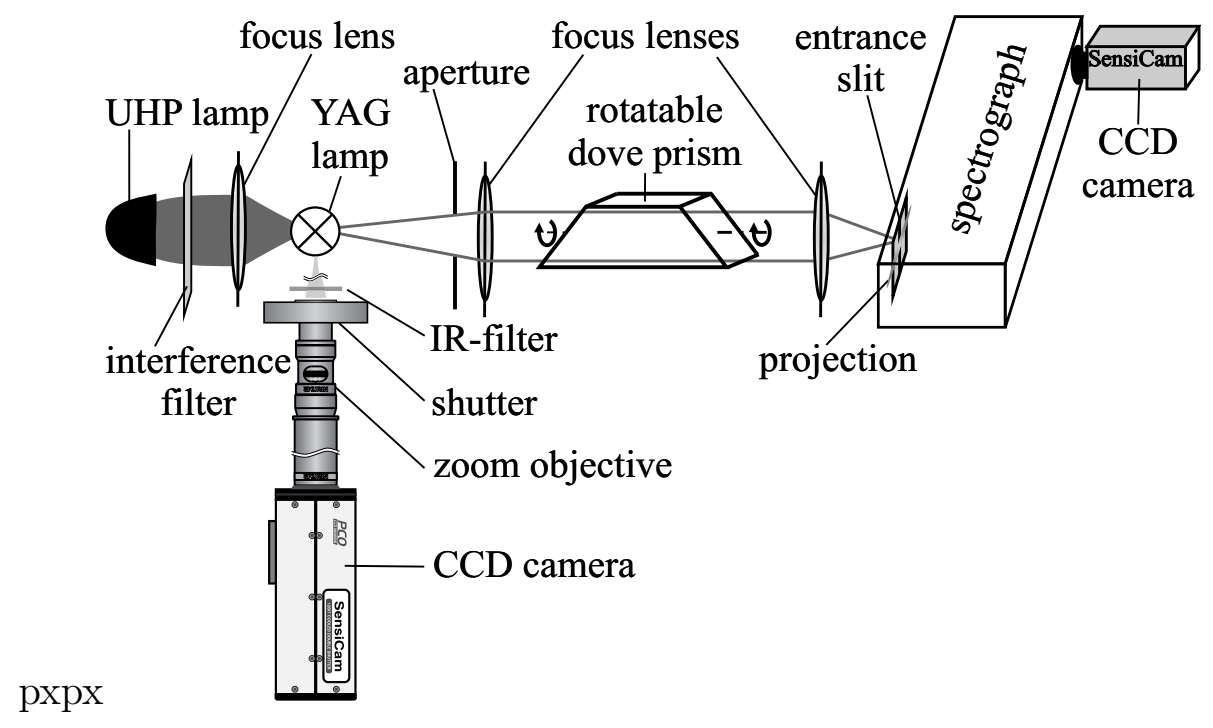

Figure 2: Schematic drawing of the complete optical set up

Elektronik DCU/I 2250-28, max. voltage: $400 \mathrm{~V}$, max. current: $28 \mathrm{~A}$, max. power: $2250 \mathrm{VA}$ ) which is controlled by a voltage from a signal generator (Agilent 33120A).

\subsection{Measuring system for phase resolved emission and absorption spectroscopy}

A schematic drawing of the optical set up is shown in figure 2. To determine the axial electrode temperature profile within the YAG lamp seeded with $\mathrm{Ce}_{3}$ the upper electrode of the YAG lamp is focused on a CCD camera ( $P C O$ Sensicam Fastshutter) by means of a zoom objective (Navitar Zoom 6000). The record is controlled by an electromechanical shutter (Uniblitz VS14) in front to avoid the undesirable smear effect [32]. A narrow bandpass filter at $890 \mathrm{~nm}(10 \mathrm{~nm}$ FWHM) between the CCD camera system and the YAG lamp is used for recording a two dimensional image at the particular wavelength. By calibration with a tungsten ribbon lamp (Wi17g3) from OSRAM absolute electrode temperatures $T_{e l}(z)$ are deduced from the camera picture. Furthermore, the shape of the arc attachment can be extracted from the images. This 2D-method was presented in [22] for the first time. By replacing the infrared filter in front of the CCD camera by a neutral density filter (Newport) it is also possible to record more detailed images of the arc attachment.

Optical emission spectroscopy is realised by a $1 / 4 \mathrm{~m}$ spectrograph (Chromex 250is, grating: 1200 lines $/ \mathrm{mm}$ ) and a second CCD camera (PCO Sensicam QE). Two lenses (focus length of $310 \mathrm{~mm}$, respectively) project a 1:1 image of the lamp on the entrance slit of the spectrograph. A rotatable so-called Dove prism between the two lenses is used for turning the image around the optical axis.

To measure the axially resolved electrode temperature $T_{e l}(z)$ spectroscopically, the upper electrode within the YAG lamp seeded with CsI has to be adjusted by means 
of the prism parallel to the entrance slit of the spectrograph (slit width: $100 \mu \mathrm{m}$ ). An edge filter at $600 \mathrm{~nm}$ is positioned in front of the entrance slit to suppress radiation from the second order spectrum produced by the grating at $\lambda_{\text {meas }} / 2$. Measurements of $T_{e l}(z)$ are performed at a single suitable wavelength in the infrared region where at best no plasma radiation is present. After a calibration with the tungsten ribbon lamp, absolute temperature values are received.

To determine the plasma temperature $T_{p l}$ and particle densities of atoms $N_{a}$ and ions $N_{i}$ in front of the electrode, the emission of a discharge cross section at a fixed distance from the electrode tip has to be captured by the entrance slit of the spectrograph (slit width: $25 \mu \mathrm{m}$ ). For this purpose the image of the upper lamp electrode is adjusted perpendicular to the slit by turning the Dove prism through $45^{\circ}$ around the optical axis. The intensities, recorded by the CCD camera at wavelengths from approximately $350 \mathrm{~nm}$ to $950 \mathrm{~nm}$, are calibrated in absolute units by means of the tungsten ribbon lamp. Radial resolved measurements of $T_{p l}, N_{a}$ and $N_{i}$ are performed in this work $125 \mu \mathrm{m}$ in front of the upper electrode. They are accomplished by an evaluation of radial distributions of line emission coefficients from measured lateral line intensity distributions by an inverse Abel transformation.

A more detailed description of the measuring system for emission spectroscopy can be found in $[14,24,26,33]$.

To measure particle densities by absorption spectroscopy the set up for emission spectroscopy is extended by a back light source being positioned behind the YAG lamp on the elongation of the optical axis $[20,21,31]$. A powerful broadband light source with a high radiance within the visible spectral range and a temporarily very stable emission is realized with an ultra high pressure (UHP) lamp [34]. The UHP lamp (Philips 228 L4), originally designed for video projection systems, is electrically driven by the electronic circuit from a video projector. It is operated with a $90 \mathrm{~Hz}$ switched-dc current. Its amplitude amounts to $2.2 \mathrm{~A}$ and the electrical power input to $120 \mathrm{~W}$. A specially designed reflector is already equipped around the UHP lamp so that a maximal light power is emitted as a quasi-parallel light beam by the lamp. To minimize the influence of the irradiation by the powerful UHP lamp on the operational conditions of the YAG lamp an optical bandpass filter (Andover Corporation $50 \mathrm{~mm}$ ) is installed between both lamps. The filtered UHP light is focused by an achromatic lens (focus length of $80 \mathrm{~mm}$ ) to an area with a diameter of $d=10 \mathrm{~mm}$ around the measuring point. It provides a largely homogeneous radiance perpendicular to axis of the YAG lamp. The beam emanating from the UHP lamp is not strictly parallel within the tube of the YAG lamp as it is indicated in figure 2. But the angle of beam spread is so small and the optical path length within the discharge so short that the beam divergence can be neglected. A calibration of the optical signals with the tungsten ribbon lamp is unnecessary due to the special analysis of the measuring data presented in section 4.2. By the same reason the phase modulation of the light emission of the UHP lamp which is on the order of $2 \%$ has not to be considered. 
All quantities are measured with some phase resolution. It corresponds to temporal resolution as long as the lamp operation is really periodic. It is accomplished by trigger signals for the cameras, which are shifted with an adjustable time delay with respect to a well defined reference phase angle. A reproducible reference phase angle can be deduced from zero crossing of the lamp voltage as it was shown by Langenscheidt [13]. To trigger the cameras only the rising edge of the lamp voltage is used. The uncertainty of the trigger pulse does not exceed $2 \mu \mathrm{s}$. A pulse delay generator (Stanford Research Systems DG 535) is used to shift the trigger signal from voltage zero crossing. A block diagram of the trigger set up can be found in [14].

\begin{tabular}{|c|c|c|c|c|}
\hline $\begin{array}{l}\text { Lamp } \\
\text { type }\end{array}$ & $\begin{array}{c}\text { Electrode } \\
\text { temperature } \\
T_{e l} \\
\end{array}$ & $\begin{array}{c}\text { Plasma } \\
\text { temperature } \\
T_{p l} \\
\end{array}$ & $\begin{array}{c}\text { Atom } \\
\text { density } \\
N_{a}\end{array}$ & $\begin{array}{c}\text { Ion } \\
\text { density } \\
N_{i} \\
\end{array}$ \\
\hline $\mathrm{Hg}$ & $\begin{array}{c}\text { emission } \\
\text { spectroscopy }\end{array}$ & $\begin{array}{c}\text { emission } \\
\text { spectroscopy }\end{array}$ & - & - \\
\hline Cs & $\begin{array}{c}\text { emission } \\
\text { spectroscopy }\end{array}$ & $\begin{array}{c}\text { emission } \\
\text { spectroscopy }\end{array}$ & $\begin{array}{c}\text { emission } \\
\text { spectroscopy }\end{array}$ & $\begin{array}{c}\text { Saha } \\
\text { equation }\end{array}$ \\
\hline $\mathrm{Ce}$ & 2D-method & $\begin{array}{c}\text { emission } \\
\text { spectroscopy }\end{array}$ & $\begin{array}{c}\text { absorption } \\
\text { spectroscopy }\end{array}$ & $\begin{array}{c}\text { emission } \\
\text { spectroscopy }\end{array}$ \\
\hline Dy & $\begin{array}{c}\text { emission } \\
\text { spectroscopy }\end{array}$ & $\begin{array}{c}\text { emission } \\
\text { spectroscopy }\end{array}$ & $\begin{array}{c}\text { emission } \\
\text { spectroscopy }\end{array}$ & $\begin{array}{c}\text { emission } \\
\text { spectroscopy }\end{array}$ \\
\hline $\mathrm{Tl} / \mathrm{Dy}$ & $\begin{array}{c}\text { emission } \\
\text { spectroscopy }\end{array}$ & $\begin{array}{c}\text { emission } \\
\text { spectroscopy }\end{array}$ & $\begin{array}{c}\text { emission } \\
\text { spectroscopy }\end{array}$ & $\begin{array}{c}\text { emission } \\
\text { spectroscopy }\end{array}$ \\
\hline
\end{tabular}

Table 3: Measuring methods used in this work and in [26] for electrode temperature $T_{e l}$, plasma temperature $T_{p l}$ and particle density measurements of atoms $N_{a}$ and ions $N_{i}$ of the respective emitter material $C s, C e$ and $D y$

In table 3 the different measuring methods used in this and in the reference work [26] are assigned to the lamp types given in table 2 .

\section{Pyrometric electrode temperature measurement $(1 \lambda$-method $)$}

For accurate pyrometric temperature measurements a segment of the spectrum emitted by the electrode has to be chosen which is as far as possible in the infrared region but still in the sensitivity range of the CCD camera. In addition the plasma radiation emitted by the YAG lamp in the selected spectral range should be insignificant compared to the emission of the electrode surface. In the case of the YAG lamp seeded with CsI the emission of the electrode in the infrared is superimposed by an intense multi line emission of $C s$ [35]. Therefore a pyrometric measurement with a spectrograph is an 
appropriate choice. An inspection of the spectrum showed that an applicable wavelength for this kind of temperature measurement is $745 \mathrm{~nm}$. But in the case of a lamp seeded with $\mathrm{CeI}_{3}$ both requirements are met within a spectral range around $890 \mathrm{~nm}$ as was checked spectroscopically. Therefore the 2D-method with an interference filter at $890 \mathrm{~nm}$ is the more favourable pyrometric measuring procedure.

The spectral radiance along the upper electrode $I_{e l}(\lambda, z)$ at $\lambda=745 \mathrm{~nm}$ or $\lambda=890 \mathrm{~nm}$ is recorded by a single measurement at a selected phase angle $\varphi$. It is assumed that the spectral radiance emitted by the tungsten electrodes is represented by that of a grey body radiator $I_{g b}\left(\lambda, T_{e l}\right)$, which can be calculated in dependence on the temperature $T_{e l}$ and wavelength $\lambda$ from Planck's law and the spectral emissivity of tungsten $\epsilon\left(\lambda, T_{e l}\right)<1$. Reliable spectral emissivities of tungsten are available in literature [36]. Axial temperature profiles $T_{e l}(z, \varphi)$ are obtained by comparing $I_{e l}(\lambda, z, \varphi)$ and $I_{g b}\left(\lambda, T_{e l}\right)$.

The surface roughness is enhanced by the arc attachment in the vicinity of the electrode tip and with it the emissivity is exaggerated in an undefined manner compared to the values given in literature. As a consequence the temperature measurement at the electrode tip becomes doubtful. In previous investigations of HID electrodes operated with a dc-current the axial electrode temperature distribution was additionally simulated by integrating the 1D heat balance of the electrode and matching it to the measured distribution within a region of confidence, in which the values of $\epsilon$ were trustable. The temperature of the electrode tip $T_{t i p}$ was then determined by an extrapolation of the integrated 1D heat balance. Reliable results were obtained with this method for electrodes operated with a dc-current $[1,6,11]$. But if the electrodes are operated with an ac-current the modulation of the electrode temperature is limited to a small area behind the electrode tip [15]. Therefore also the tip region with its exaggerated values of $\epsilon$ has to be taken into account when the integral of the heat balance is matched to the measured temperature distribution. A more realistic temperature profile can be used for the matching procedure if instead of a one dimensional a two dimensional temperature distribution is available. In this case it is easier to identify and remove locally excessive temperatures. A detailed discussion of the limited measuring accuracy of $T_{\text {tip }}$ is presented in [15, 26]. It was shown that the phase resolved experimental determination of $T_{t i p}$ is afflicted with an error of the order of $\Delta T_{t i p} \approx 50 \mathrm{~K}$.

\section{Spectroscopic plasma temperature and particle density measurements}

\subsection{Emission spectroscopy}

The spectral radiances $I_{\nu}(y)$ of optical thin lines are measured in a fixed distance $d$ from the electrode tip along a $y$-axis perpendicular to the optical axis $x$ and the discharge 
axis $z$. They are calibrated in absolute units and integrated over line profiles, providing lateral distributions of line intensities $I_{L}(y)$. By an inverse Abel transformation of $I_{L}(y)$ radial emission coefficients $\epsilon_{L}(r)$ are obtained if the discharge is rotational symmetric.

\begin{tabular}{ll}
\hline$\lambda_{1}=576.96 \mathrm{~nm}$ & $\lambda_{2}=579.07 \mathrm{~nm}$ \\
\hline$A_{1}=1.709 \cdot 10^{8} \mathrm{~s}^{-1}$ & $A_{2}=2.039 \cdot 10^{8} \mathrm{~s}^{-1}$ \\
$g_{1}=5$ & $g_{2}=5$ \\
$E_{1}=8.853 \mathrm{eV}$ & $E_{2}=8.845 \mathrm{eV}$ \\
\hline
\end{tabular}

Table 4: Spectroscopic data for the calculation of the plasma temperature $T_{p l}$ from Hg-line intensities [38]

At first the plasma temperature $T_{p l}$ inside a YAG lamp is determined using two overlapping mercury lines at $\lambda_{1}=576.96 \mathrm{~nm}$ and $\lambda_{2}=579.07 \mathrm{~nm}[15,24,37]$. The corresponding spectroscopic data is given in table 4 with the transition probabilities $A_{1}$ and $A_{2}$, the statistical weights $g_{1}$ and $g_{2}$ and the excitation energies of the upper line levels $E_{1}$ and $E_{2}$. By assuming local thermal equilibrium, the common temperature of all particles, in this paper always named as plasma temperature $T_{p l}$, is calculated from the sum of the respective line emission coefficients postulating a Boltzmann population of the upper emitting states and using the ideal gas law.

$$
\epsilon_{u l}=\frac{h c_{0}}{4 \pi} \frac{p_{0}}{k_{b} T_{p l}} \frac{1}{Q_{H g}\left(T_{p l}\right)}\left[\frac{g_{1} A_{1}}{\lambda_{1}} \exp \left(-\frac{E_{1}}{k_{b} T_{p l}}\right)+\frac{g_{2} A_{2}}{\lambda_{2}} \exp \left(-\frac{E_{2}}{k_{b} T_{p l}}\right)\right]
$$

A constant mercury pressure of $p_{0}=2 \mathrm{MPa}$ is assumed. A plasma temperature of $T_{p l} \leq 7000 \mathrm{~K}$ with an error margin of about $4 \%$ was found within YAG lamps with similar parameters using the two $\mathrm{Hg}$-lines [24, 26].

The $C s$ atom density $N_{a, C s}$ within the YAG lamp seeded with $C s I$ and the $C e$ ion density $N_{i, C e}$ within the lamp seeded with $C e I_{3}$ are evaluated by means of $\epsilon_{L}(r)$ of the respective atomic or ionic line, assuming a Boltzmann population of the emitting states defined by $T_{p l}(r)$. In both cases two overlapping lines are used. The sum of their emission coefficients is given by a formula as that for the two mercury lines (equation 1 ) in which the factor $p_{0} / k_{b} T_{p l}$ is exchanged by $N_{a, C s}$ or $N_{i, C e}$. The corresponding spectroscopic data for a determination of $N_{a, C s}$ is presented in table 5 while the data for the evaluation of $N_{i, C e}$ is given in table 6 .

The complete measuring and data processing procedure is described in detail in $[24,26,31,33,39]$.

The determination of the densities $N_{a, C s}$ of $C s$ atoms and $N_{i, C e}$ of $C e$ ions by emission spectroscopy is afflicted mainly with three different error sources: the 
Investigating the gas phase emitter effect of Cs and Ce in ceramic metal halide lamps11

\begin{tabular}{ll}
\hline$\lambda_{1}=697.33 \mathrm{~nm}$ & $\lambda_{2}=698.35 \mathrm{~nm}$ \\
\hline$A_{1}=7.514 \cdot 10^{6} \mathrm{~s}^{-1}$ & $A_{2}=1.296 \cdot 10^{6} \mathrm{~s}^{-1}$ \\
$g_{1}=6$ & $g_{2}=4$ \\
$E_{1}=3.232 \mathrm{eV}$ & $E_{2}=3.230 \mathrm{eV}$ \\
\hline
\end{tabular}

Table 5: Spectroscopic data for the calculation of $C s$ atom density $N_{a, C s}$ [38]

\begin{tabular}{ll}
\hline$\lambda_{1}=457.23 \mathrm{~nm}$ & $\lambda_{2}=457.28 \mathrm{~nm}$ \\
\hline$A_{1}=2.663 \cdot 10^{7} \mathrm{~s}^{-1}$ & $A_{2}=1.515 \cdot 10^{7} \mathrm{~s}^{-1}$ \\
$g_{1}=12$ & $g_{2}=10$ \\
$E_{1}=3.395 \mathrm{eV}$ & $E_{2}=4.192 \mathrm{eV}$ \\
\hline
\end{tabular}

Table 6: Spectroscopic data for the calculation of $C e$ ion density $N_{i, C e}$ [38]

measuring inaccuracy of the emission coefficient $\epsilon_{u l}$, which amounts at least $20 \%$, the uncertainty of the transition probabilities of at least $10 \%$ and the measuring error of the plasma temperature $T_{p l}$ of $4 \%$, which is multiplied by a factor $E_{u} / k_{b} T_{p l}$ being on the order of $5-7$. The error sources are discussed in more detail in [26]. The resulting error margins are quite high, they are at least on the order of $50 \%$. They show that the particle densities obtained by emission spectroscopy represent only an order of magnitude, but the variations in dependence on phase, $\partial N_{a} / \partial \varphi$ and $\partial N_{i} / \partial \varphi$, provide a quite reliable information.

\subsection{Absorption spectroscopy}

The resulting radiance $I_{\nu}^{r e s}(y)$ composed of the radiance emitted by the UHP lamp $I_{\nu}^{a b s, U H P}(y)$ and of the radiance emitted by the YAG lamp $I_{\nu}^{e m, Y A G}(y)$ is measured, when both lamps are operated simultaneously, in addition to the radiance emitted by the YAG lamp $I_{\nu}^{e m, Y A G}(y)$ alone. The difference between the two radiances

$$
I_{\nu}^{a b s, U H P}(y)=I_{\nu}^{r e s}(y)-I_{\nu}^{e m, Y A G}(y)=I_{\nu}^{U H P}(y) \exp \left[-\int_{x=-R / 2}^{x=+R / 2} \alpha(\nu, x, y) d x\right]
$$

provides the absorption of the UHP back light $I_{\nu}^{U H P}(y)$ by the lamp plasma being given by the integral over the frequency-dependent local absorption coefficient $\alpha(\nu, \vec{r})$ along the optical path within the lamp, called optical depth $\tau$. $I_{\nu}^{a b s, U H P}(y)$ is recorded in a distance $z=d$ from the electrode tip along the lateral coordinate $y . R$ is the discharge radius. A plot of $I_{\nu}^{a b s, U H P}$ in dependence on $\lambda$ for a fixed value of $y$ may show a dip corresponding to the profile of the absorbing line. The undisturbed intensity of the UHP back light $I_{\nu}^{U H P}(y)$ is not measured separately but determined by an interpolation of the course of $I_{\nu}^{U H P}(y)$ between the wings of the absorption dip. An example showing a dip being formed by a $C e$ atom resonance line emitted by the YAG lamp seeded with 
Investigating the gas phase emitter effect of Cs and Ce in ceramic metal halide lamps12

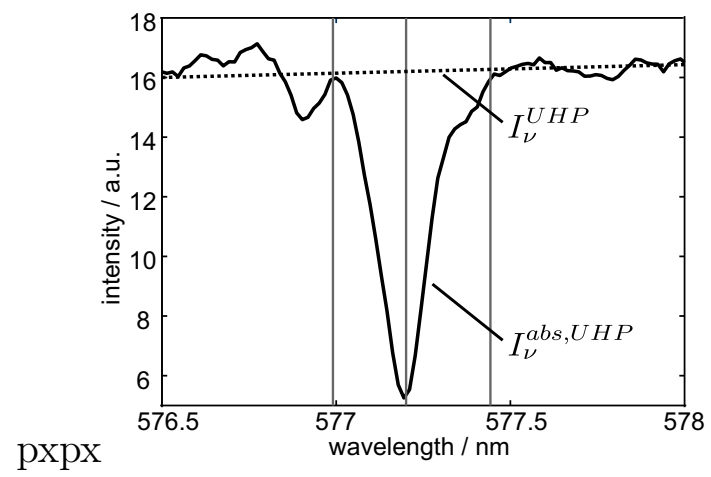

Figure 3: Example for the absorbed $I_{\nu}^{a b s, U H P}(-)$ and interpolated $I_{\nu}^{U H P}(\ldots)$ UHP back light at a $C e$ atom resonance line at $\lambda=577.36 \mathrm{~nm}$ measured $125 \mu \mathrm{m}$ in front of the upper electrode. Parameter: $\mathrm{CeI}_{3}$, electrode diameter $d_{E}=0.36 \mathrm{~mm}$, electrode length $l_{E}=5 \mathrm{~mm}, i=0.8 \mathrm{~A}$ switched-dc

$\mathrm{CeI}_{3}$ and the extrapolation of $I_{\nu}^{U H P}$ is given in figure 3. The advantage of the fitting procedure is a proportion $I_{\nu}^{U H P}(y) / I_{\nu}^{a b s, U H P}(y)$, which is independent of fluctuations of the UHP back light and does not depend on the properties of the YAG lamp tube and their changes by temperature effects [20].

The optical depth $\tau(\nu, y)$ is calculated according to

$$
\tau(\nu, y)=\int_{x=-R / 2}^{x=+R / 2} \alpha(\nu, x, y) d x=\ln \left(\frac{I_{\nu}^{U H P}(y)}{I_{\nu}^{a b s, U H P}(y)}\right) .
$$

By an integration of the absorption coefficient $\alpha(\nu)$ with respect to the frequency $\nu$ a so called line absorption coefficient $\alpha_{L}$ is defined:

$$
\alpha_{L}=\int_{\Delta \nu} \alpha(\nu) d \nu=N_{l} \frac{e^{2}}{4 \pi \epsilon_{0}} \frac{\pi}{m_{e} c_{0}} f_{l u} .
$$

$N_{l}$ is the population density of the absorbing atomic state and $f_{l u}$ the absorption oscillator strength of the specific optical transition, the other symbols have the usual meaning. $\alpha_{L}$ can be related to the measured absorption by an integration of the optical depth $\tau$ over a frequency interval $\Delta \nu$, which comprehends the absorption dip. By an inverse Abel transformation of

$$
\int_{\Delta \nu} \tau(\nu, y) d \nu=\int_{\Delta \lambda} \tau(\lambda, y) \frac{c_{0}}{\lambda^{2}} d \lambda=\int_{x=-R / 2}^{x=+R / 2} \alpha_{L}(x, y) d x
$$

the radial line absorption coefficient $\alpha_{L}(r)$ is obtained and with it the radial density distribution $N_{l}(r)$ of the absorbing atomic state if the discharge is rotational symmetric.

If $N_{l}$ is the ground state of an atomic resonance line the total atomic density $N_{a}(r)$ can be calculated from:

$$
N_{a}(r)=N_{l}(r) \frac{Q_{a}\left(T_{p l}\right)}{g_{l}}=\frac{\alpha_{L}(r)}{f_{l u}} \frac{4 \pi \epsilon_{0}}{e^{2}} \frac{m_{e} c_{0}}{\pi} \frac{Q_{a}\left(T_{p l}\right)}{g_{l}} .
$$


Investigating the gas phase emitter effect of Cs and Ce in ceramic metal halide lamps13

$g_{l}$ is the statistical weight of the atomic ground state and $Q_{a}$ the corresponding atomic partition function.

$$
\begin{aligned}
& \hline \lambda=577.36 \mathrm{~nm} \\
& \hline f=5.492 \cdot 10^{-3} \\
& g_{0}=9 \\
& \hline
\end{aligned}
$$

Table 7: Spectroscopic data of the $C e$ resonance line [38] for the calculation of $C e$ atom density $N_{a, C e}$

The $C e$ resonance line at $\lambda=577.36 \mathrm{~nm}$ is chosen to determine the $C e$ atom density $N_{a, C e}(r)$ within the YAG lamp seeded with $\mathrm{CeI}_{3}$. An interference filter with a centre wavelength of $577 \mathrm{~nm}$ and a bandwidth of $10 \mathrm{~nm}$ FWHM is used to avoid a distracting irradiation of the YAG lamp by the UHP lamp. The atom density $N_{a, C e}(r)$ is evaluated from $\alpha_{L}$ taking into account the spectroscopic data of table 7 and the partition function $Q_{a}\left(T_{p l}\right)$ of the $C e$ atoms. It is calculated using the atomic data given in [38]. The absorption by the wings of the mercury lines at $576.96 \mathrm{~nm}$ and $579.07 \mathrm{~nm}$ is eliminated by using the extrapolated value of $I_{\nu}^{U H P}$ as a reference.

As an example $N_{a, C e}(r)$ obtained by a measurement at a switched-dc current of $0.8 \mathrm{~A}$ at $10 \mathrm{~Hz}$ within the cathodic phase at phase angle $\varphi=7 \pi / 5$ is presented in figure 4.

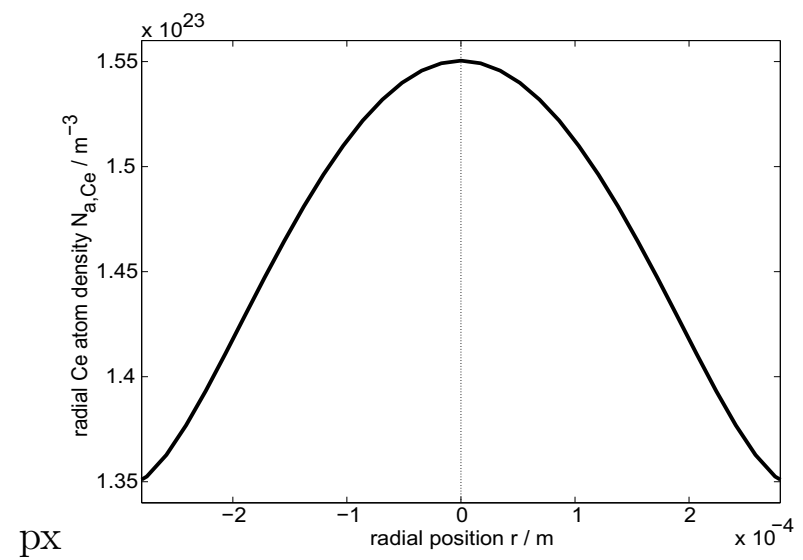

Figure 4: Radial $C e$ atom density $N_{a, C e}(r)$ measured $125 \mu \mathrm{m}$ in front of the upper electrode. Parameter: $\mathrm{CeI}_{3}$, electrode diameter $d_{E}=0.36 \mathrm{~mm}$, electrode length $l_{E}=5 \mathrm{~mm}, i=0.8 \mathrm{~A}$ switched-dc, $10 \mathrm{~Hz}, \varphi=7 \pi / 5$ (cathodic phase) 
Investigating the gas phase emitter effect of Cs and Ce in ceramic metal halide lamps14

The logarithmic derivative of equation 6 is used to estimate the error margins of $N_{a, C e}$ :

$$
\left|\frac{\Delta N_{a, C e}}{N_{a, C e}}\right|=\left|\frac{\Delta \alpha_{L}}{\alpha_{L}}\right|+\left|\frac{\Delta f_{l u}}{f_{l u}}\right|+\left|\frac{\partial \ln Q_{a, C e}}{\partial \ln T_{p l}} \frac{\Delta T_{p l}}{T_{p l}}\right| .
$$

The line absorption coefficient $\alpha_{L}$ may be afflicted with an error of the order of $20 \%$ mainly by reason of the inverse Abel transformation, which reacts, especially on the axis, quite sensitive on deviations from symmetry of the lateral distribution of the integrated optical depth. The knowledge of the oscillator strength $f_{l u}$ is not better than $10 \%$. The error margin of the plasma temperature, which is multiplied in equation 7 by $\partial \ln Q_{a, C e} / \partial \ln T_{p l} \approx 1$, is of the order of $4 \%$. The resulting error margin is of the order of $\left|\Delta N_{a, C e} / N_{a, C e}\right| \cdot 100 \approx 35 \%$, which is much lower than the error margins of the particle densities determined by emission spectroscopy. This means that the absorption spectroscopy provides, different to the emission spectroscopy, quantitative information on particle densities within the YAG lamp.

A first discussion of the broadband absorption spectroscopy with an UHP lamp is given in $[20,31]$ while first results by means of this method are presented for YAG lamps seeded with $\mathrm{DyI}_{3}$ in $[21,31]$ and with $\mathrm{CeI}_{3}$ in $[31,40]$.

\section{Estimation of the $C s$ ion density by SAHA equation}

A spectroscopic measurement of the $C s$ ion density $N_{i, C s}$ within the YAG lamp seeded with $C s I$ is nearly impossible, since the energy gap between the ion ground state and the lowest excited state, which emits lines in the visible spectral range $\left(\lambda_{1,2,3,4}=\right.$ $492.57,522.70,527.40,537.10 \mathrm{~nm}[41])$, is very large. It amounts to $E_{i, e x}=15.68 \mathrm{eV}$, so that its population and with it the intensity of the ion lines is extremely low for plasma temperatures of the order of $7000 \mathrm{~K}$ in the lamp. Alternatively $N_{i, C s}$ may be evaluated by inserting the $C s$ atom density $N_{a, C s}$ and the plasma temperature $T_{p l}$ into the SAHA equation for caesium $S_{C s}\left(T_{p l}\right)$, using the fact that LTE is established at the measuring point within the lamp plasma:

$$
\begin{aligned}
& S(T)=\frac{N_{i, C s} N_{e}}{N_{a, C s}} \\
& =\frac{Q_{i, C s}\left(T_{p l}\right)}{Q_{a, C s}\left(T_{p l}\right)} \frac{2\left(2 \pi m_{e} k_{b} T_{p l}\right)^{3 / 2}}{h^{3}} \exp \left(-\frac{E_{i, C s}}{k_{b} T_{p l}}\right) .
\end{aligned}
$$

But this raises the question whether the electron density $N_{e}$ is equal to the $C s$ ion density $N_{i, C s}$. The ionisation energy of caesium $\left(E_{i, C s}=3.893 \mathrm{eV}\right)$ is much lower than the ionisation energy of iodine $\left(E_{i, I}=10.45 \mathrm{eV}\right)$ and of mercury $\left(E_{i, H g}=10.43 \mathrm{eV}\right)$. It is clear that the iodine ion density $N_{i, I}$ can be neglected compared to the $C s$ ion density $N_{i, C s}$, if is taken into account that the corresponding atom densities within the ratio of the SAHA equations for iodine and caesium are of the same order of magnitude:

$$
\frac{N_{i, I}}{N_{i, C s}}=\frac{N_{a, I}}{N_{a, C s}} \frac{Q_{i, I} Q_{a, C s}}{Q_{a, I} Q_{i, C s}} \exp \left(-\frac{E_{i, I}-E_{i, C s}}{k_{b} T_{p l}}\right) .
$$


But the density of $\mathrm{Hg}$ atoms, $N_{a, H g}$, is three orders of magnitude higher than the density of $I$ atoms so that the density of the $H g$ ions $N_{i, H g}$ may be comparable to that of the $C s$ ions $N_{i, C s}$, as is shown by a relation similar to equation 9 , in spite of a much lower ionisation degree of mercury compared to caesium.

Due to quasi neutrality the electron density $N_{e}$ is determined in the YAG lamp filled with $H g$ and $C s I$ by

$$
N_{e} \approx N_{i, C s}+N_{i, H g}
$$

Combining equation 10 and the SAHA equations for caesium $S_{C s}\left(T_{p l}\right)$ (equation 8) and that for mercury $S_{H g}\left(T_{p l}\right)$, the ion densities $N_{i, C s}$ and $N_{i, H g}$ can be expressed by the corresponding atom densities according to:

$$
\begin{aligned}
N_{i, C s} & =\frac{S_{C s}\left(T_{p l}\right) N_{a, C s}}{\left[S_{H g}\left(T_{p l}\right) N_{a, H g}+S_{C s}\left(T_{p l}\right) N_{a, C s}\right]^{1 / 2}} \\
N_{i, H g} & =\frac{S_{H g}\left(T_{p l}\right) N_{a, H g}}{\left[S_{C s}\left(T_{p l}\right) N_{a, C s}+S_{H g}\left(T_{p l}\right) N_{a, H g}\right]^{1 / 2}} .
\end{aligned}
$$

Results for $N_{i, C s}$ and $N_{i, H g}$ will be given in chapter 6.1. Equation 11 shows that the $C s$ ion density $N_{i, C s}$ is reduced by the competing ionisation of $\mathrm{Hg}$. According to equation 11 the error margins of $N_{i, C s}$ may be still higher than those of the $C s$ atom density $N_{a, C s}$ :

$$
\frac{\Delta N_{i, C s}}{N_{i, C s}} \geq \frac{\Delta N_{a, C s}}{N_{a, C s}}
$$

\section{Results and discussion of measurements at the YAG lamps}

Measurements are performed at YAG lamps which are operated with switched-dc current of $0.8 \mathrm{~A}$ and operating frequencies of $1,10,100,500 \mathrm{~Hz}$ and $1 \mathrm{kHz}$. At most, measurements at 10 equidistant phase angles over one period are accomplished. For operating frequencies greater than $100 \mathrm{~Hz}$ it was often not possible to carry out more than one measurement per half period. In the subsequent figures, which present the phase resolved measuring results, the first half period $(\varphi=0-\pi)$ is the anodic and the second half period $(\varphi=\pi-2 \pi)$ the cathodic phase. Phase resolved results of the electrode tip temperature $T_{t i p}(\varphi)$, plasma temperature $T_{p l}(\varphi)$ and density of atoms $N_{a}(\varphi)$ and ions $N_{i}(\varphi)$ are presented. The pyrometric measurements are performed at the upper electrode, all spectroscopic measurements at a distance of $125 \mu \mathrm{m}$ in front of it. The reported values of $T_{p l}(\varphi), N_{a}(\varphi)$ and $N_{i}(\varphi)$ are averages over a rotational symmetric cross section with the same diameter as the electrode. 


\subsection{YAG lamp seeded with CsI}

Figure 5 shows the electrode tip temperature $T_{t i p}(\varphi)$ of the upper electrode for different frequencies. The maximum values of $T_{t i p}(\varphi)$ are at least $290 \mathrm{~K}$ below the melting point of tungsten of $3695 \mathrm{~K}$. The courses of $T_{\text {tip }}(\varphi)$ demonstrate for $f<100 \mathrm{~Hz}$ a heating of the electrode within the anodic phase while in the cathodic phase a reduction of its heating is visible. The modulation diminishes with increasing frequency, but the resulting average value of $T_{\text {tip }}(\varphi)$ increases with frequency for frequencies of $f \geq 500 \mathrm{~Hz}$. It is obvious that a reduction of $T_{t i p}(\varphi)$ of at least $200 \mathrm{~K}$ is induced by the $C s$ atoms and $C s$ ions within the discharge compared to a YAG lamp with $H g$ filling only being shown in figure 1(a) of the reference paper [26]. It amounts even $400 \mathrm{~K}$ for $f=1 \mathrm{kHz}$. But the increase of $T_{t i p}(\varphi)$ with increasing frequency is a striking difference to the decrease of $T_{\text {tip }}(\varphi)$ in YAG lamps seeded with rare earth iodides as is shown in [26] figure 5(a) for $D y I_{3}$ and in section 6.2 for $\mathrm{CeI}_{3}$.

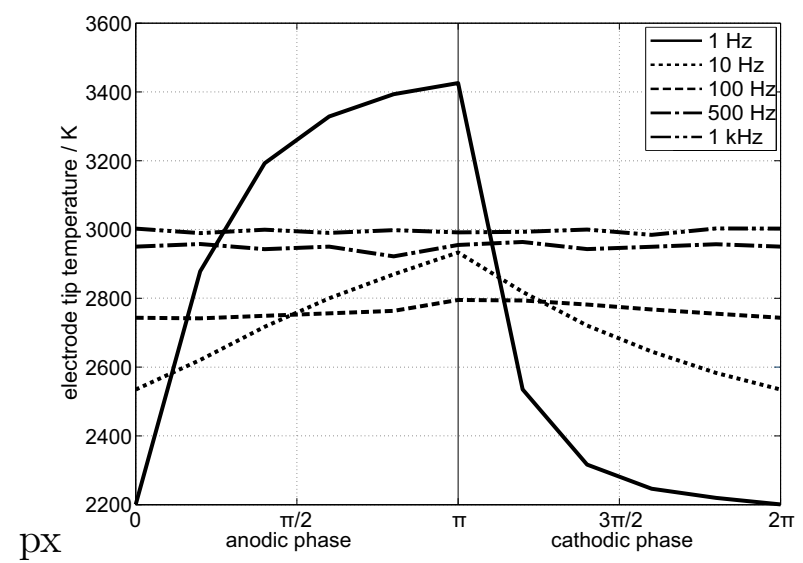

Figure 5: Electrode tip temperature $T_{\text {tip }}(\varphi)$ measured at the upper electrode over one period for various frequencies. Parameter: CsI, electrode diameter $d_{E}=0.36 \mathrm{~mm}$, electrode length $l_{E}=5 \mathrm{~mm}, i=0.8 \mathrm{~A}$ switched-dc

Figure 6(a) presents the plasma temperature $T_{p l}(\varphi)$ measured in front of the upper electrode for different frequencies. $T_{p l}(\varphi)$ is at least $700 \mathrm{~K}$ higher in the anodic phase than in the cathodic phase. $T_{p l}(\varphi)$ decreases in the anodic phase for $f \geq 500 \mathrm{~Hz}$. The considerably reduced plasma temperature in front of the cathode indicates an enhancement of the $C s$ density within the cathodic phase by cataphoresis. It is generated by the electric force on the $C s^{+}$ions and a counteracting pressure gradient of the $C s$ ion density. The density of the $C s$ ions and $C s$ atoms is coupled by charge exchange, ionisation and recombination processes. A flow of $C s$ atoms, which is propelled by a decline of $C s$ atoms in front of the cathode, compensates the $C s$ ion current towards the cathode. By reason of the low ionisation energy $E_{i}=3.89 \mathrm{eV}$ of $C s$ only a low plasma temperature is required to generate a sufficiently high electron density $N_{e}$ in front of the cathode. Similar courses of $T_{p l}(\varphi)$ were measured by Langenscheidt et al at a YAG 
lamp seeded with $\mathrm{NaI}, \mathrm{TlI}$ and $\mathrm{DyI}_{3}$ [24]. The plasma temperature in front of the upper electrode $T_{p l}(\varphi)$ of a YAG lamp without any salt filling ([26] figure 2) or seeded with $\mathrm{CeI}_{3}$ (figure $8(\mathrm{~b})$ ) shows a completely different behaviour for the same current amplitude and waveform. $T_{p l}(\varphi)$ is generally higher in these cases, moreover it is higher in the cathodic phase than in the anodic phase. In addition with increasing frequency a decrease of $T_{p l}(\varphi)$ independent of the phase is visible. Hence it is obvious that the ionisation energy $E_{i}$ of the respective metal iodide has a distinct influence on $T_{p l}(\varphi)$.

The $H g$ ion density $N_{i, H g}(\varphi)$, estimated with equation 12, is shown for different frequencies in figure $6(\mathrm{~b})$. The course of $N_{i, H g}(\varphi)$ is similar to the course of $T_{p l}(\varphi)$ (figure 6(a)): $N_{i, H g}(\varphi)$ is up to 18 times higher within the anodic phase than within the cathodic phase where $N_{i, H g}(\varphi)$ varies between $2 \cdot 10^{20} \mathrm{~m}^{-3}$ and $4.5 \cdot 10^{21} \mathrm{~m}^{-3} \cdot N_{i, H g}(\varphi)$ decreases simultaneously with $T_{p l}(\varphi)$ in the anodic phase for $f \geq 500 \mathrm{~Hz}$. The course of $N_{i, H g}(\varphi)$ reflects the influence of the mercury ionisation on $N_{e}$ particularly in the anodic phase. Hence $N_{i, H g}(\varphi)$ has to be taken into account referred to equation 10 when the $C s$ ion density $N_{i, C s}(\varphi)$ is determined with SAHA equation.

Figure $7\left(\right.$ a) shows the $C s$ atom density $N_{a, C s}(\varphi)$ determined in front of the upper electrode for different frequencies. $N_{a, C s}(\varphi)$ is lower by one order of magnitude in the anodic phase than in the cathodic phase. $N_{a, C s}(\varphi)$ increases weakly in the anodic phase with increasing frequency and decreases by one order of magnitude in the cathodic phase. For higher operating frequencies $(f \geq 500 \mathrm{~Hz})$ the density is nearly constant over time.

The $C s$ ion density $N_{i, C s}(\varphi)$ being estimated with equation 11 is shown for different frequencies in figure $7(\mathrm{~b})$. The values of $N_{i, C s}(\varphi)$ are higher than the $C s$ atom density $N_{a, C s}(\varphi)$ which is caused by the low ionisation energy $E_{i}$ of $C s$, but the trend is nearly the same. $N_{i, C s}(\varphi)$ is according to $N_{a, C s}(\varphi)$ in the anodic phase by one order of magnitude lower than in the cathodic phase. $N_{i, C s}(\varphi)$ decreases in the cathodic phase and increases in the anodic phase with increasing frequency, except for $f=1 \mathrm{kHz}$. Also an assimilation of $N_{i, C s}(\varphi)$ takes place with increasing frequency within both phases.

The increase of $N_{a, C s}(\varphi)$ (figure $\left.7(\mathrm{a})\right)$ and $N_{i, C s}(\varphi)$ (figure $7(\mathrm{~b})$ ) in the cathodic phase demonstrates the existence of a $C s$ ion current towards the cathode. It may not only substitute in part the thermionic electron emission of the cathode but above all produce a monolayer of $C s$ atoms on the surface of the cathode. This layer may reduce the work function and thereby the temperature of the electrode. According to numerical calculations given in [4] the reduction of $T_{t i p}(\varphi)$ is only marginally induced by a lowering of the plasma temperature $T_{p l}(\varphi)$ caused by the $C s$ vapour in front of the cathode, but mainly by a reduction of the work function $\phi$ by a $C s$ monolayer. Its formation becomes obvious by comparing the electrode tip temperatures $T_{\text {tip }}(\varphi)$ in figure 5 and figure 1 (a) of [26]. Furthermore, the missing formation of an emitter spot within the cathodic phase is an indication for a moderate $C s$ ion current density in front of the cathode $[20,24]$. The decrease of $N_{a, C s}(\varphi)$ and $N_{i, C s}(\varphi)$ in the anodic phase is caused by the 
reversal of the $C s$ ion current. But this takes place with a finite time constant, as is indicated by the increase of the $C s$ atom and $C s$ ion density in front of the anode with increasing frequency. Nevertheless figure 5 reveals an increase of the average electrode tip temperature with increasing frequency. This may result from a decreasing phase averaged coverage of the electrode surface with emitter atoms for increasing frequencies due to the low adsorption energy $E_{a}$ of $C s$ of $3.37 \mathrm{eV}$ (table 1). Therefore an expansion of the emitter effect to the anodic phase with increasing frequency, which may effect a reduction of the average work function, does not take place.

\subsection{YAG lamp seeded with $\mathrm{CeI}_{3}$}

Figure $8\left(\right.$ a) shows $T_{\text {tip }}(\varphi)$ of the upper electrode for different frequencies. Similar to the course of $T_{\text {tip }}(\varphi)$ of a YAG lamp seeded with CsI (figure 5) a heating of the electrode in the anodic phase and a reduction of its heating in the cathodic phase takes place. This change with polarity diminishes with increasing frequency. Additionally the maximal values of $T_{t i p}(\varphi)$ given in figure $8(\mathrm{a})$ are at least $390 \mathrm{~K}$ below the melting point of tungsten. A comparison with $T_{t i p}(\varphi)$ of a YAG lamp with $H g$ filling only (given in figure 1(a) of reference [26]) confirms a general reduction of $T_{t i p}(\varphi)$ of at least $300 \mathrm{~K}$ effected by the presence of $C e$ atoms and $C e$ ions in the arc plasma. By comparing the courses of $T_{\text {tip }}(\varphi)$ of figure 5 and $8(\mathrm{a})$ it is visible that for 1,10 and $100 \mathrm{~Hz}$ the temperature values of the YAG lamp seeded with $C s I$ are below $T_{t i p}(\varphi)$ of the lamp seeded with $\mathrm{CeI}_{3}$, only for $1 \mathrm{~Hz}$ between $\varphi=\pi / 2$ and $\varphi=\pi$ a more pronounced temperature increase is observed for a lamp seeded with CsI. But the most important difference to figure 5 is a decrease of $T_{\text {tip }}(\varphi)$ with increasing frequency. For $f=1 \mathrm{kHz}$ a reduction of $\Delta T_{t i p}(\varphi)=600 \mathrm{~K}$ is induced by a seeding with $C e I_{3}$ compared to a YAG lamp operated with a pure mercury vapour atmosphere.

$T_{p l}(\varphi)$, measured in front of the upper electrode, is presented in figure 8(b) for different frequencies. The values of $T_{p l}(\varphi)$ decrease slightly with increasing frequency. $T_{p l}(\varphi)$ is higher in the cathodic phase than in the anodic phase. Similar courses of $T_{p l}(\varphi)$ were measured by Langenscheidt et al at a YAG lamp seeded only with $D y I_{3}$, beeing shown in figure 12 and figure 13 of reference [24]. It reflects a higher power requirement to generate $C e$ ions instead of $C s$ ions by reason of the higher ionisation energy of $C e$. At $1 \mathrm{kHz}$ the courses of $T_{p l}(\varphi)$ in the anodic and cathodic phase converge. $T_{p l}(\varphi)$ is at most $150 \mathrm{~K}$ higher in comparison to a YAG lamp with $\mathrm{Hg}$ filling only ([26] figure 2) while the course of $T_{p l}(\varphi)$ is similar. The difference between the plasma temperatures in both lamps is within the error margin of approximately $300 \mathrm{~K}$.

Figure 9(a) shows courses of the $C e$ atom density $N_{a, C e}(\varphi)$ while figure $9(\mathrm{~b})$ represents courses of the $C e$ ion density $N_{i, C e}(\varphi)$ in front of the upper electrode for different frequencies. The $C e$ atom density as well as the $C e$ ion density is higher in the cathodic than in the anodic phase. But the difference of the $C e$ atom density 
within the cathodic and anodic phase is distinctly smaller than in YAG lamps seeded with $C s I$ (figure $7(\mathrm{a})$ ) or with $\mathrm{DyI}_{3}$ (as is shown in figure 3 and figure 4 of reference [26]). Moreover the $C e$ atom density $N_{a, C e}(\varphi)$ and ion density $N_{i, C e}(\varphi)$ are higher than the corresponding $C s$ densities. Especially they are of the order of ten higher than the $D y$ atom and ion densities (being shown in figure 3 of reference [26]). Both $C e$ densities decrease with increasing frequency in the cathodic phase. But in the anodic phase a general trend is not visible for the $C e$ atom density $N_{a, C e}(\varphi)$ as well as for the $C e$ ion density $N_{i, C e}(\varphi) . N_{i, C e}(\varphi)$ increases within the cathodic phase with a time constant on the order of $10 \mathrm{~ms}$ and $N_{a, C e}(\varphi)$ with an extended time constant of $20 \mathrm{~ms}$. Accordingly at operating frequencies above several ten Hertz the maximum values of $N_{a, C e}(\varphi)$ and $N_{i, C e}(\varphi)$ are lowered with increasing frequency. The variation of $N_{i, C e}(\varphi)$ and the delayed variation of $N_{a, C e}(\varphi)$ in dependence on phase and on operating frequency demonstrates that $C e$ is transported cataphoreticly in front of the electrode. A $C e$ atom monolayer is deposited on the electrode surface by a $C e$ ion current from the plasma to the cathode. It reduces the work function and therefore $T_{t i p}(\varphi)$ which is proved by comparing figure 8(a) with figure 1(a) of [26]. The reduction of $N_{a, C e}(\varphi)$ and $N_{i, C e}(\varphi)$ in the anodic phase is produced by a reversal of the $C e$ ion current. $N_{a, C e}(\varphi)$ and $N_{i, C e}(\varphi)$ are nearly constant over time for higher frequencies indicating that the $C e$ ions are too immobile to follow the frequency above $500 \mathrm{~Hz}$. They remain directly in front of the electrode, recombine and form a layer of $C e$ atoms on the electrode surface even during the anodic phase. Thus the emitter effect remains active also within the anodic phase and because of this $T_{t i p}(\varphi)$ decreases with increasing frequency (figure $8(\mathrm{a})$ ).

\section{Conclusion}

In the YAG lamp seeded with $C s I$ a cathodic gas phase emitter effect due to a $C s$ ion current is observed at low frequencies $(f<500 \mathrm{~Hz})$, but the emitter effect does not overlap onto the anodic phase with increasing frequency. This is caused by an insufficient coverage of the electrode surface with $C s$ atoms within the anodic phase due to the low adsorption energy $E_{a}$ of $C s\left(E_{a}=3.37 \mathrm{eV}\right)$ within the anodic phase. It leads to an increase of the averaged electrode tip temperature $T_{t i p}(\varphi)$ with increasing frequency. A direct indication that the layer of $C s$ atoms is removed within the anodic phase is the increase of $T_{\text {tip }}(\varphi)$ at the end of the anodic phase for $f=1 \mathrm{~Hz}$ up to $3400 \mathrm{~K}$, which exceeds the otherwise higher electrode tip temperature $T_{\text {tip }}(\varphi)$ of a YAG lamp seeded with $\mathrm{CeI}_{3}$ by $100 \mathrm{~K}$. Though, the cathodic gas phase emitter effect reduces the work function and temperature of the electrode and increases the lifetime of the lamp.

In the YAG lamp seeded with $\mathrm{CeI}_{3}$ a gas phase emitter effect is produced at low frequencies $(f<500 \mathrm{~Hz})$ mainly by a $C e$ ion current in the cathodic phase whereas it disappears with a time constant of some milliseconds within the anodic phase. The reduction of the electrode tip temperature $T_{t i p}(\varphi)$ is at low frequencies less pronounced than within a YAG lamp seeded with $C s I$ but more pronounced than within a lamp seeded with $D y I_{3}$. The difference between the $C e$ and $C s$ lamp can be attributed to the 
lower work function $\phi$ of the $C s$ monolayer compared to that of $C e$, but the difference between the $C e$ and $D y$ lamp can be attributed, at least in part, to the higher adsorption energy $E_{a}$ of $C e$ compared to $D y$ on tungsten. The higher adsorption energy $E_{a}$ of $C e$ may also be the reason for a higher $C e$ atom and ion density in front of the electrode and a weaker modulation of the $C e$ particle density in dependence on polarity compared to $D y$. Moreover it has to be taken into account that the spectroscopic measurements at a distance of $125 \mu \mathrm{m}$ from the electrode tip reflect only partially the density variation immediately in front of the electrode.

At higher operating frequencies the reduction of the electrode tip temperature $T_{\text {tip }}(\varphi)$ is most pronounced within the $C e$ lamp, less distinct within the Dy lamp and even reversed within the $C s$ lamp. It reflects the decrease of the adsorption energy $E_{a}$ from $C e$ to $D y$ and $C s$ which corresponds to a successive reduction of the life time of the $C e, D y$ and $C s$ monolayer. The emitter effect and with it the reduction of the electrode tip temperature overlaps to the anodic phase at higher frequencies with increasing life time of the monolayer, e.g. for lamps seeded with $C e I_{3}$ or $D y I_{3}$ but not for lamps seeded with $C s I$ in spite of an increase of the $C s$ ion density in front of the anode with increasing frequency.

The findings are another test of the hypothesis that in HID lamps emitter material can be deposited on the surface of hot tungsten electrodes by an ion current from the arc plasma to the cathode. Appropriate materials are able to reduce the work function of tungsten by the formation of an atomic monolayer and with it the electrode temperature.

It supplements the investigation of the gas phase emitter effect of $D y$ given in a preceding paper [26]. In this paper not only a correlation between an enhanced $D y$ ion density in front of the electrode and a reduced electrode temperature was demonstrated. It was also confirmed by an additional seeding of the YAG lamp with $T l I$ that not the substitution of thermionic electron emission by an ion current but a reduced work function is responsible for the temperature reduction of the electrode. The vapour pressure of $T l I$ is much higher than that of $\mathrm{DyI}_{3}$. The metallic $\mathrm{Tl}$ exhibits a much higher work function than metallic $D y$, but the ionisation energy of $T l$ vapour $\left(E_{i}=6.11 \mathrm{eV}\right)$ is nearly the same as that of $D y$ vapour. Therefore the current transferred by metal ions in front of the cathode within a lamp additionally seeded with $T l I$ cannot be smaller than in a lamp seeded only with $D y I_{3}$. In spite of that the emitter effect is suppressed by the thallium additive in accordance with its higher work function.

\section{Acknowledgements}

The authors thank Philips Lighting, NL, for scientific and financial support. Financial support from the Research School of the Ruhr-University Bochum is also gratefully acknowledged by the authors. 
Investigating the gas phase emitter effect of Cs and Ce in ceramic metal halide lamps21

\section{References}

[1] Dabringhausen L et al 2002 J. Phys. D: Appl. Phys. 35 1621-1630

[2] Nandelstaedt D et al 2002 J. Phys. D: Appl. Phys. 35 1639-1647

[3] Almanstoetter J et al 2002 J. Phys. D: Appl. Phys. 35 1751-1756

[4] Benilov M S et al 2005 J. Phys. D: Appl. Phys. 38 3155-62

[5] Benilov M S 2008 J. Phys. D: Appl. Phys. 41144001

[6] Redwitz M et al 2006 J. Phys. D: Appl. Phys. 39 2160-2179

[7] Mentel J and Heberlein J 2010 J. Phys. D: Appl. Phys. 43023002

[8] Hartmann T et al 2010 J. Phys. D: Appl. Phys. 43025201

[9] Lichtenberg S et al 2002 J. Phys. D: Appl. Phys. 35 1648-1656

[10] Lichtenberg S et al 2005 J. Phys. D: Appl. Phys. 38 3112-3127

[11] Dabringhausen L et al 2005 J. Phys. D: Appl. Phys. 38 3128-3142

[12] Benilov M S et al 2002 J. Phys. D: Appl. Phys. 35 1-15

[13] Langenscheidt O et al 2007 J. Phys. D: Appl. Phys. 40 415-431

[14] Reinelt J et al 2008 J. Phys. D: Appl. Phys. 41144002

[15] Reinelt J et al 2011 J. Phys. D: Appl. Phys. 44095204

[16] Waymouth J F 1971 Electric Discharge Lamps (Monograph in Modern Electrical Technology) (Cambridge, MA.: MIT Press)

[17] Langmuir I 1914 Phys. Rev. 4544

[18] Brattain W A and Becker J A 1933 Phys. Rev. 43 428-450

[19] Luhmann J et al 2002 J. Phys. D: Appl. Phys. 35 1631-1638

[20] Westermeier M et al 2010 J. Phys. D: Appl. Phys. 43124015

[21] Westermeier M et al 2010 Phase resolved Dy-density and plasma temperature measurements by absorption- and emision-spectroscopy of Dy spectral lines, Proc. 12th Int. Symp. on the Science and Technology of Light Sources and the 3rd Int. Conf. on White LEDs and Solid State Lighting (Eindhoven (NL), 11-16 July 2010) ed Haverlag M et al CP060, 165-166

[22] Luijks G M J F et al 2005 J. Phys. D: Appl. Phys. 38 3163-3169

[23] Tielemans P and Oostvogels F 1983 Philips J. Res. 38 214-223

[24] Langenscheidt O et al 2008 J. Phys. D: Appl. Phys. 41144005

[25] Luijks G M J F et al 2008 J. Phys. D: Appl. Phys. 41144006

[26] Reinelt J et al 2011 J. Phys. D: Appl. Phys. 44224006

[27] Alton G D 1986 Surface Science 175 226-240

[28] Miedema A R and Dorleijn J W F 1980 Surface Science 95 447-464

[29] Alekseev N I 1968 Soviet Physics - Technical Physics 12 (12) 1639-1642

[30] Medvedev B K, Ionov N I and Belyakov Yu I 1974 Sov. Phys. Solid State 15 (9) 1743-1746

[31] Westermeier M 2011 Investigation of the emitter effect in HID-lamps by absorption-and emissionspectroscopy, PhD thesis, Ruhr-Universität Bochum

[32] PCO AG 2008 pco.knowledgebase: smear v. 0710

[33] Reinelt J 2009 Experimental investigation on electrodes of HID lamps at low and high operating frequencies, PhD thesis, Ruhr-Universität Bochum

[34] Derra G et al 2005 J. Phys. D: Appl. Phys. 38 2995-3010

[35] Pichler G et al 2003 Physica Scripta T105 98-100

[36] DeVos J C 1954 Physica 20 690-714

[37] Schoepp H 2002 Elektrische und strahlungsphysikalische Eigenschaften von Hochdruckentladungen - insbesondere Quecksilberdampfentladungen - für die Beleuchtungstechnik (Berlin: Technische Universität)

[38] Kurucz R et al 1995 KURUCZ CD-ROM NO. 23 - Atomic Line List (Cambridge, MA: HarvardSmithsonian Center for Astrophysics)

[39] Langenscheidt O 2008 Elektroden für HID-Lampen - Diagnostik und Simulation, PhD thesis, RuhrUniversität Bochum 
Investigating the gas phase emitter effect of Cs and Ce in ceramic metal halide lamps22

[40] Ruhrmann C et al 2010 Determination of the ground state density of Ce in front of the electrode of a YAG lamp by absorption spectroscopy, Proc. 12th Int. Symp. on the Science and Technology of Light Sources and the 3rd Int. Conf. on White LEDs and Solid State Lighting (Eindhoven (NL), 11-16 July 2010) ed Haverlag M et al CP152, 445-446

[41] Striganov A R and Sventitskii N S 1968 Tables of spectral lines of neutral and ionized atoms (IFI/PlenumDataCorporation, New York) 
Investigating the gas phase emitter effect of Cs and Ce in ceramic metal halide lamps23

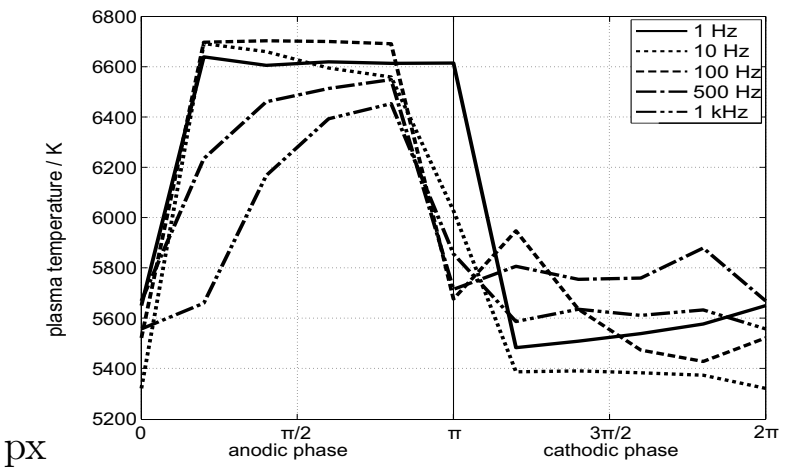

(a) Plasma temperature

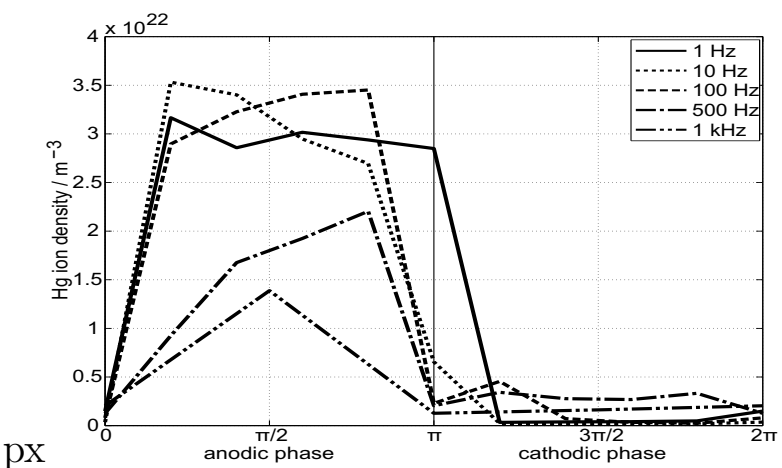

(b) $\mathrm{Hg}$ ions (SAHA equation)

Figure 6: Plasma temperature $T_{p l}(\varphi)$ and mercury ion density $N_{i, H g}(\varphi)$ at a distance of $125 \mu \mathrm{m}$ in front of the upper electrode over one period for various frequencies. Parameter: $C s I$, electrode diameter $d_{E}=0.36 \mathrm{~mm}$, electrode length $l_{E}=5 \mathrm{~mm}$, $i=0.8$ A switched-dc

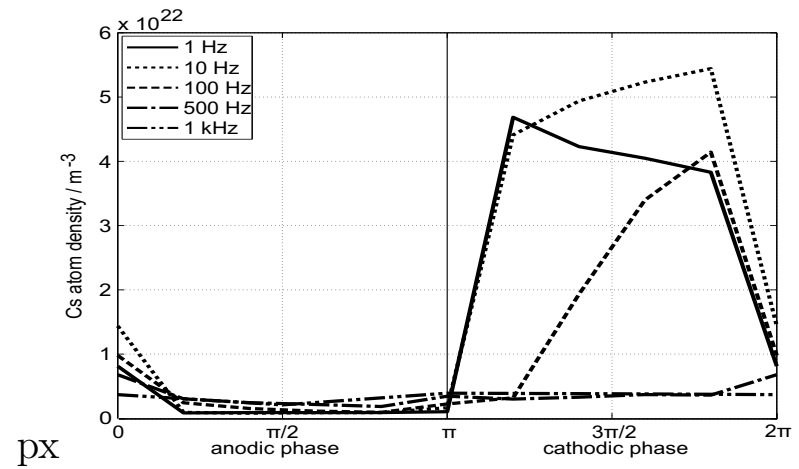

(a) $C s$ atoms

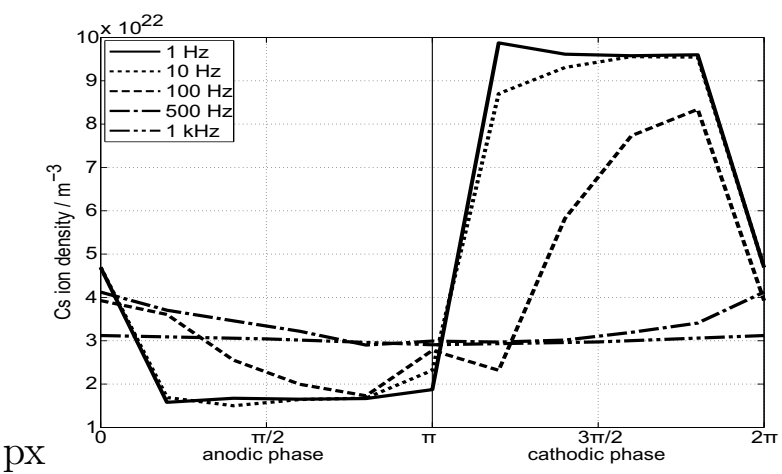

(b) $C s$ ions (SAHA equation)

Figure 7: Caesium atom density $N_{a, C s}(\varphi)$ and estimated ion density $N_{i, C s}(\varphi)$ over one period for various frequencies at a distance of $125 \mu \mathrm{m}$ in front of the upper electrode. Parameter: $C s I$, electrode diameter $d_{E}=0.36 \mathrm{~mm}$, electrode length $l_{E}=5 \mathrm{~mm}$, $i=0.8$ A switched-dc 
Investigating the gas phase emitter effect of Cs and Ce in ceramic metal halide lamps24

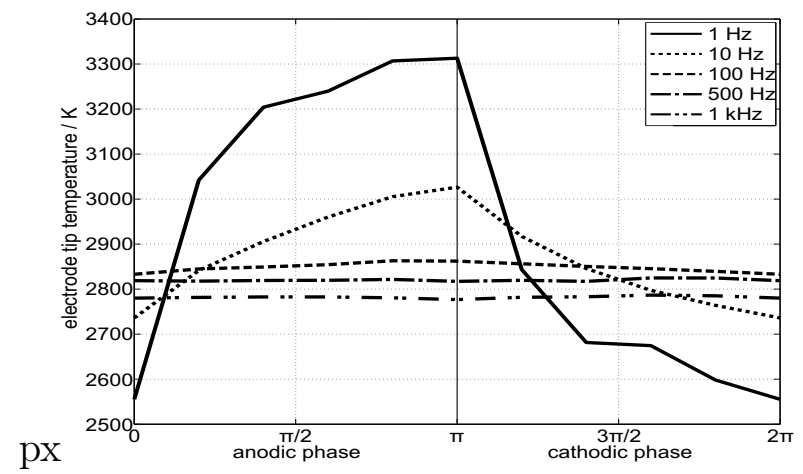

(a) Electrode tip temperature

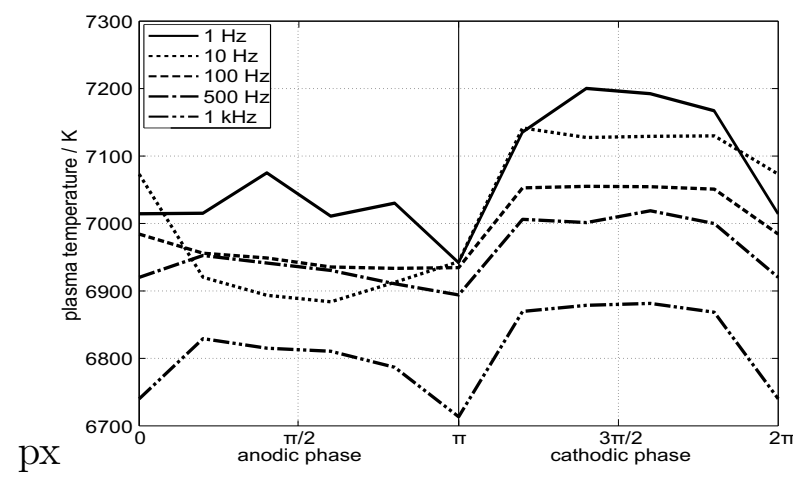

(b) Plasma temperature

Figure 8: Electrode tip temperature $T_{t i p}(\varphi)$ measured at the upper electrode over one period for various frequencies and plasma temperature $T_{p l}(\varphi)$ measured over one period for various frequencies at a distance of $125 \mu \mathrm{m}$ in front of the upper electrode. Parameter: $C e I_{3}$, electrode diameter $d_{E}=0.36 \mathrm{~mm}$, electrode length $l_{E}=5 \mathrm{~mm}, i=0.8$ A switched$\mathrm{dc}$

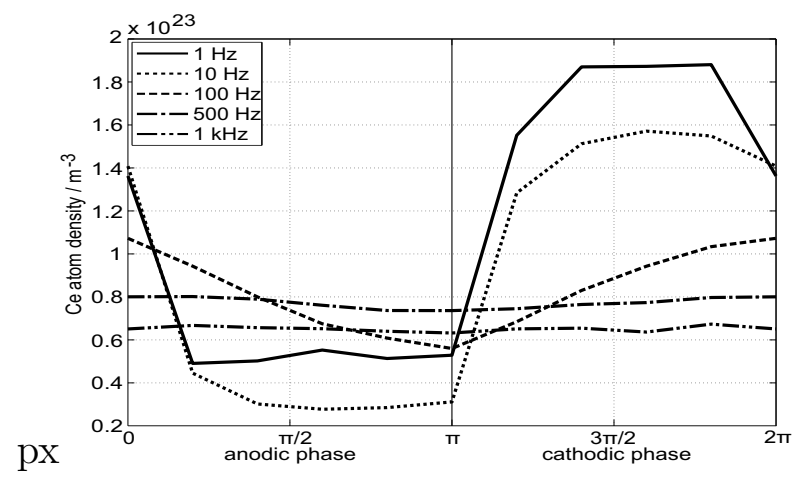

(a) Ce atoms

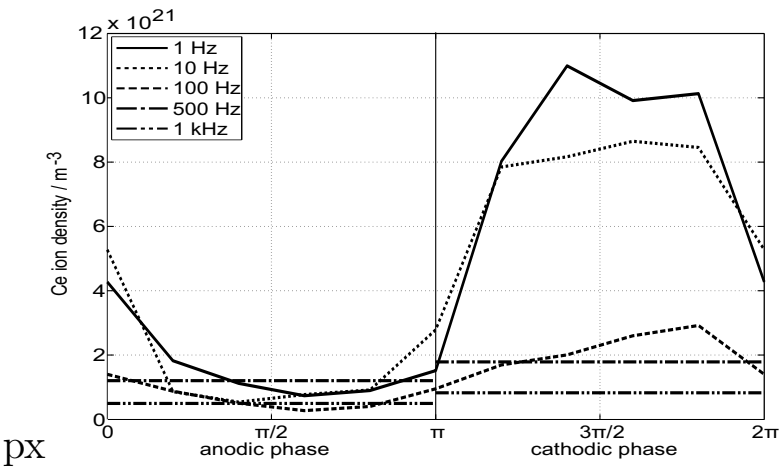

(b) $\mathrm{Ce}$ ions

Figure 9: Cerium atom density $N_{a, C e}(\varphi)$ and ion density $N_{i, C e}(\varphi)$ over one period for various frequencies at a distance of $125 \mu \mathrm{m}$ in front of the upper electrode. Parameter: $C e I_{3}$, electrode diameter $d_{E}=0.36 \mathrm{~mm}$, electrode length $l_{E}=5 \mathrm{~mm}, i=0.8 \mathrm{~A}$ switched$\mathrm{dc}$ 